\title{
Quantification of cytoskeletal deformation in living cells based on hierarchical feature vector matching.
}

Citation for published version (APA):

Delhaas, T., van Engeland, S., Broers, J. L. V., Bouten, C., Kuijpers, N., Ramaekers, F. C. S., \& Snoeckx, L. H. E. M. (2002). Quantification of cytoskeletal deformation in living cells based on hierarchical feature vector matching. American Journal of Physiology-Cell Physiology, 283, C639-C645.

https://doi.org/10.1152/ajpcell.00535.2001

Document status and date:

Published: 01/01/2002

DOI:

10.1152/ajpcell.00535.2001

Document Version:

Publisher's PDF, also known as Version of record

\section{Please check the document version of this publication:}

- A submitted manuscript is the version of the article upon submission and before peer-review. There can be important differences between the submitted version and the official published version of record.

People interested in the research are advised to contact the author for the final version of the publication, or visit the DOI to the publisher's website.

- The final author version and the galley proof are versions of the publication after peer review.

- The final published version features the final layout of the paper including the volume, issue and page numbers.

Link to publication

\footnotetext{
General rights rights.

- You may freely distribute the URL identifying the publication in the public portal. please follow below link for the End User Agreement:

www.umlib.nl/taverne-license

Take down policy

If you believe that this document breaches copyright please contact us at:

repository@maastrichtuniversity.nl

providing details and we will investigate your claim.
}

Copyright and moral rights for the publications made accessible in the public portal are retained by the authors and/or other copyright owners and it is a condition of accessing publications that users recognise and abide by the legal requirements associated with these

- Users may download and print one copy of any publication from the public portal for the purpose of private study or research.

- You may not further distribute the material or use it for any profit-making activity or commercial gain

If the publication is distributed under the terms of Article $25 \mathrm{fa}$ of the Dutch Copyright Act, indicated by the "Taverne" license above, 


\section{Quantification of cytoskeletal deformation in living cells based on hierarchical feature vector matching Tammo Delhaas, Saskia van Engeland, Jos Broers, Carlijn Bouten, Nico Kuijpers, Frans Ramaekers and Luc H. E. H. Snoeckx \\ Am J Physiol Cell Physiol 283:639-645, 2002. First published Mar 27, 2002; \\ doi:10.1152/ajpcell.00535.2001}

You might find this additional information useful...

Supplemental material for this article can be found at:

http://ajpcell.physiology.org/cgi/content/full/283/2/C639/DC1

This article cites 16 articles, 11 of which you can access free at: http://ajpcell.physiology.org/cgi/content/full/283/2/C639\#BIBL

Updated information and services including high-resolution figures, can be found at: http://ajpcell.physiology.org/cgi/content/full/283/2/C639

Additional material and information about AJP - Cell Physiology can be found at: http://www.the-aps.org/publications/ajpcell

This information is current as of March 3, 2005 . 


\title{
special communication
}

\section{Quantification of cytoskeletal deformation in living cells based on hierarchical feature vector matching}

\author{
TAMMO DELHAAS, ${ }^{1,2}$ SASKIA VAN ENGELAND, ${ }^{4}$ JOS BROERS,${ }^{4,3}$ CARLIJN BOUTEN,${ }^{4}$ \\ NICO KUIJPERS, ${ }^{4}$ FRANS RAMAEKERS ${ }^{3}$ AND LUC H. E. H. SNOECKX ${ }^{1,4}$ \\ Departments of ${ }^{1}$ Physiology, ${ }^{2}$ Pediatrics, and ${ }^{3}$ Molecular Cell Biology, \\ Maastricht University, 6200 MD Maastricht; and ${ }^{4}$ Faculty of Biomedical \\ Engineering, Eindhoven University of Technology, 5512 AZ Eindhoven, The Netherlands
}

Received 13 November 2001; accepted in final form 7 March 2002

Delhaas, Tammo, Saskia van Engeland, Jos Broers, Carlijn Bouten, Nico Kuijpers, Frans Ramaekers, and Luc H. E. H. Snoeckx. Quantification of cytoskeletal deformation in living cells based on hierarchical feature vector matching. Am J Physiol Cell Physiol 283: C639-C645, 2002. First published March 27, 2002; 10.1152/ajpcell.00535. 2001.-The cytoskeleton is a dynamic scaffold in living cells even in the absence of externally imposed forces. In this study on cytoskeletal deformation, the applicability of hierarchical feature vector matching (HFVM), a new matching method, currently applied in space research and three-dimensional surface reconstruction, was investigated. Stably transfected CHO-K1 cells expressing green fluorescent protein (GFP) coupled to vimentin were used to visualize spontaneous movement of the vimentin cytoskeleton of individual cells using a confocal laser scanning system. We showed that, with proper parameter and configuration settings, HFVM could recognize and trace $60-70 \%$ of all image points in artificially translated, rotated, or deformed images. If only points belonging to the cytoskeleton were selected for matching purposes, the percentage of matched points increased to $98 \%$. This high percentage of recognition also could be reached in a time series of images, in which a certain degree of bleaching of the fluorescence over the recording time of 30 min was inevitable. In these images, HFVM allowed the detection as well as the quantification of spontaneous cytoskeletal movements of up to $10 \%$ of the cell width. Therefore, HFVM appears to be a reliable method of quantifying dynamic cytoskeletal behavior in living cells.

vimentin; fibroblast; green fluorescent protein; confocal laser scanning

VARIOUS CELL TYPES are sensitive to mechanical loading. For instance, cardiac myocytes immediately increase their contractile force upon increased load. Vascular

Address for reprint requests and other correspondence: T. Delhaas, Depts. of Pediatrics and Physiology, Cardiovascular Research Institute Maastricht, Maastricht Univ. and University Hospital Maastricht, PO Box 5800, 6202 AZ Maastricht, The Netherlands (E-mail: t.delhaas@fys.unimaas.nl). endothelial cells respond to changes in blood flow, in particular to shear stress forces, with the release of nitric oxide, prostaglandins, and related molecules. This leads to relaxation or contraction of the underlying smooth muscle cells and, thus, the regulation of vascular tonus $(4,5)$. Furthermore, chronic mechanical loading leads to changes in cellular gene expression, which is the prelude for structural remodeling and adaptation $(9,16)$. To activate the transcription of genes within the nucleus of the cell, the mechanical signal has to be transmitted throughout the cell. Upon mechanical loading, cells respond by activating an intracellular cascade of molecular transmitters, followed by transcriptional, synthetic, and structural changes (2-4). The mechanism by which the cellular responses are evoked remains unclear. It might be that cell surfacedeforming forces generate local biochemical responses, evoke responses by a mechanically transduced signal to the cell nucleus via the cytoskeleton, or evoke cellular responses by a combination of both mechanisms (4).

To obtain insight into the (physical) mechanisms involved in cellular responses to deformation, quantitative information of the dynamic behavior of the cytoskeleton and its response to external loading is necessary. Although fluorescent microspheres attached to the cellular surface $(1,10)$ have been used to quantify cellular deformation in response to external loading, these studies have yielded very limited spatial information. Moreover, with the use of this technique, no information can be obtained regarding intracellular/ cytoskeletal deformation. Recently, green fluorescent protein (GFP)-vimentin fusion proteins expressed in cells were used for the visualization of changes in the position of the intermediate filament cytoskeleton $(8$,

The costs of publication of this article were defrayed in part by the payment of page charges. The article must therefore be hereby marked "advertisement" in accordance with 18 U.S.C. Section 1734 solely to indicate this fact. 
14, 18). However, these studies were limited to the calculation of displacements of vimentin dots and end points of vimentin filaments. In contrast, in the present study, we will present hierarchical feature vector matching (HFVM), a robust image analysis algorithm that can be used for all sorts of applications, such as three-dimensional (3-D) surface reconstruction (12), navigation of spacecraft or autonomous robots (13), the visualization of weather satellite images, and, as described here, the quantification of the deformation of the entire vimentin cytoskeleton up to maximal physiological deformation. HFVM thereby provides a tool for the evaluation of the effects of various loading regimes on cytoskeletal deformation. Once this deformation is known, it can be coupled to molecular cellular responses, thereby providing insight into the nature of the relation between mechanical loading and cellular responses.

\section{MATERIALS AND METHODS}

Cell culture and constructs. Stably transfected CHO-K1 cells expressing GFP coupled to vimentin were used. For generation of the construct, human vimentin cDNA, kindly provided by Dr. C. Sommers (Medical Breast Cancer Section, National Cancer Institute, National Institutes of Health, Bethesda, MD), was ligated in-frame into the pS65T-C 1 vector (Clontech, Palo Alto, CA). CHO-K1 cells were transfected using DOTAP (Roche Diagnostics, Mannheim, Germany), and positive clones were selected using G-418 (GIBCO Life Technologies, Paisley, UK). Stable, cloned populations of GFP-vimentin-expressing cells were obtained by visual selection of single-cell colonies after limited dilutions. Cells were seeded at low densities on coverslips placed in plastic tissue culture disks and supplied with Ham's F-12 medium containing $10 \%$ fetal calf serum. Cells were grown at $37^{\circ} \mathrm{C}$ in a humidified atmosphere of $5 \% \mathrm{CO}_{2}$ for 2 days. Subsequently, they were cultured overnight at $30^{\circ} \mathrm{C}$ to warrant proper GFP folding. To be viewed, the coverslips were placed upside down on an object slide with a circular excavation filled with medium and $2 \mathrm{mM}$ HEPES. This preparation was totally sealed with nail varnish. Upon visualization, recordings of the cytoskeleton were made at room temperature during a period of $<1 \mathrm{~h}$, after which time the cells were discarded.

Confocal microscopy. The spontaneous movement of the vimentin cytoskeleton in individual cells was visualized using a Bio-Rad MRC-600 confocal laser scanning system (BioRad, Hemel Hempstead, UK) mounted on a Zeiss Axiophot upright microscope (Carl Zeiss, Jena, Germany). The objective used was a $\times 63$ oil-immersion, 1.4 NA lens. The argon ion laser excitation was set at $488 \mathrm{~nm}$, and emission was recorded using 510- to 540-nm band-pass filters. The system settings were adjusted to produce a minimal pixel size of 0.13 $\mu \mathrm{m}$. Over a period of $30 \mathrm{~min}, 312$-D images, obtained at the central level of the cell, were recorded with an image interval of $60 \mathrm{~s}$ and saved for later deformation analysis.

Cytoskeletal deformation quantification. For the reconstruction of the cytoskeletal deformation between two successive images, HFVM, a new matching method currently only applied for matching satellite images, was employed [Joanneum Research Institute, Graz, Austria $(12,13)]$. To distinguish between two successive images, the first was designated the reference image $(r)$, and the second, the search image $(s)$. Matching was performed by computing the best corresponding search pixel for each reference pixel. From the results, a disparity map was constructed, representing the differences in coordinates between the reference pixels and the corresponding search pixels.

Instead of using an areal correlation method to solve the local matching problem, HFVM relies on a combination of local features, each of them characterizing the surrounding of each pixel in a different way. As such, in the following context a feature of a pixel is a value that numerically describes the neighborhood of that particular pixel. The 12 features used here can be described as, or approximated by, means of convolutions (see APPENDIX). The features chosen represent the local variance, the local (weighted) average, an edge detector, and the first-and second-order derivatives in horizontal and vertical direction. The feature values of each pixel were combined in one vector, the feature vector $\vec{f}$, with the $l$ th component of this vector denoted by $f_{l}$. The feature vector of each pixel of the reference image was compared with feature vectors of pixels within the search image. The amount of similarity between a reference vector and a search vector was computed by calculating the feature vector distance between the two vectors. The feature vector distance was defined such that each component of the vectors was weighted. Denoting the weight of feature $l$ as $w_{l}$, and $m$ as the number of features, the feature vector distance between the vectors $\overrightarrow{\boldsymbol{f}}$ and $\overrightarrow{\boldsymbol{g}}$ was defined as

$$
|\overrightarrow{\boldsymbol{f}}-\overrightarrow{\boldsymbol{g}}|=\sqrt{\frac{\sum_{l=1}^{m}\left[\left(f_{l}-g_{l}\right) \cdot w_{l}\right]^{2}}{\sum_{l=1}^{m} w_{l}^{2}}}
$$

For a point $p$ in the reference image with reference feature vector $\overrightarrow{\boldsymbol{r}}(p)$, computing the distance between $\overrightarrow{\boldsymbol{r}}(p)$ and each feature vector in the search image is in general too timeconsuming. Therefore, it was assumed that the matching pixel should be within a certain search area, $\sigma_{p}$, surrounding the coordinates of the reference pixel. Then, for a given pixel $p$ in the reference image, best correspondence was found at position $q$ in the search area, where the distance between $\overrightarrow{\boldsymbol{r}}(p)$ and the search feature vector $\overrightarrow{\boldsymbol{s}}(q)$ was minimal, in that

$$
|\overrightarrow{\boldsymbol{r}}(p)-\overrightarrow{\boldsymbol{s}}(q)|=\min _{\boldsymbol{f} \in \sigma_{p}}|\overrightarrow{\boldsymbol{r}}(p)-\overrightarrow{\boldsymbol{f}}|
$$

If this minimum feature vector distance exceeded a given threshold, the correspondence was judged to be invalid and the disparity for the reference pixel remained undefined. After identification of the best search vector, linear interpolation based on the neighbor feature vector distances was used to obtain a disparity vector with subpixel accuracy. Finally, errors were removed and undefined disparities were interpolated. Note that in homogeneous areas of the image, feature values of neighboring search pixels may be similar, and thus feature distances may also be similar. To overcome the problem of finding several search pixels having the same minimum feature distance, a large number of features, of which some had a larger window (e.g., 19 pixels), were used.

To improve the robustness and efficiency of the matching algorithm and to reduce the computing time of matching algorithms, search areas were limited in size by generating pyramids of the input images. The base of the pyramid contained the original image. Computing the average gray level of four pixels in a square and storing this value as one pixel in a new image created the second level. For an image of $512 \times 512$ pixels, we found that 4 iterations, thus creating 
extra pyramid levels, were sufficient for optimal matching. Matching started at the top level of the pyramid with, due to the low spatial resolution, large search areas for each pixel. The estimated disparity map was masked, excluding pixels with a low value of local variance for matching, and filtered. Undefined disparities were interpolated. The resulting disparity map was used to define the centers of the search areas for the matching procedure of the next lower level of the pyramid.

Finally, to ascertain that the estimated disparities from the reference to the search image were of reliable quality, backmatching, i.e., inverse matching from the search to the reference image, was performed as well. A match was considered invalid when the difference between the two calculated disparities exceeded a preset limit, which was normally in the order of one pixel.

Testing the HFVM program. To evaluate the setting for each feature, the weight of the features, the number of pyramid levels, and the settings for masking, filtering, and interpolation of the disparity map that yielded the best results, a $435 \times 280$-pixel reference image $r$ was manipulated in three ways: 1) a vertical translation over a distance of 20 pixels, creating image $s_{1} ; 2$ ) a counterclockwise rotation over $10^{\circ}$ with the center of the image as pivot, creating image $s_{2}$; and 3 ) an artificial deformation comparable with $10 \%$ stretching in horizontal direction and 5\% stretching in vertical direction with the center of the image remaining at the same place creating image $s_{3}$ (Fig. 1). The reference image was matched either to itself or to images $s_{1}, s_{2}$, and $s_{3}$. Because the direction and magnitude of translation, rotation, and deformation were exactly known, the matching results could be verified by calculating the root mean square error (RMSE)

$$
\operatorname{RMSE}=\sqrt{\frac{\sum\left(x_{\exp }-x_{\mathrm{match}}\right)^{2}+\left(y_{\exp }-y_{\mathrm{match}}\right)^{2}}{n}}
$$

in which $x_{\exp }$ and $y_{\exp }$ were the expected coordinates, $x_{\text {match }}$ and $y_{\text {match }}$ were the coordinates as estimated by the matching procedure, and $n$ was the number of analyzed image points.

\section{RESULTS}

Program settings. Matching the reference image with itself was performed in various ways to find optimal program settings (Fig. 1, $A$ and $B$ ). The percentage of matched points in the two images highly depended on these program settings. When just eight features, one pyramid level, and no masking, no median filtering, and no interpolation of the disparity map were used, only $20 \%$ of the pixels could be matched, with a

Fig. 1. Images of green fluorescent protein (GFP)-vimentin-transfected cell. $A$ : reference image $r ; B-E$ : matching results after application of hierarchical feature vector matching (HFVM). The reference imager was matched either to itself $(B)$ or to artificially manipulated reference images $s_{1}$ (vertical translation over a distance of 20 pixels) $(C), s_{2}$ (counterclockwise rotation over $10^{\circ}$ with the center of the image as pivot) $(D)$, or $s_{3}$ (artificial deformation comparable with $10 \%$ stretching in horizontal and 5\% stretching in vertical direction with the center of the image remaining at the same place) $(E)$. Crosses indicate no displacement, whereas arrows indicate size, direction, and origin of displacement. Only results in a $20 \times$ 20 grid are shown. If a match for a grid point is not defined, the grid was translated 10 points, either horizontally or vertically.
A

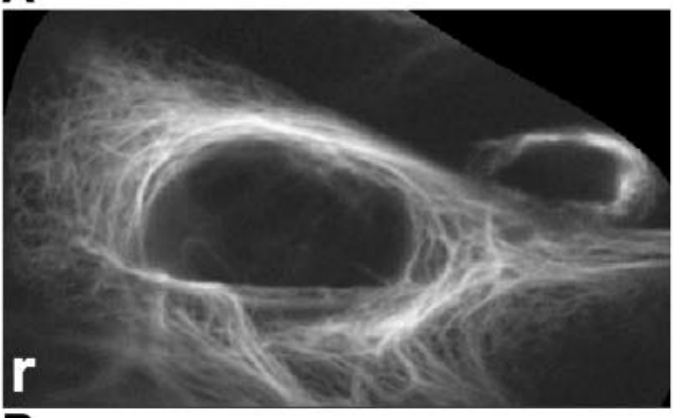

B

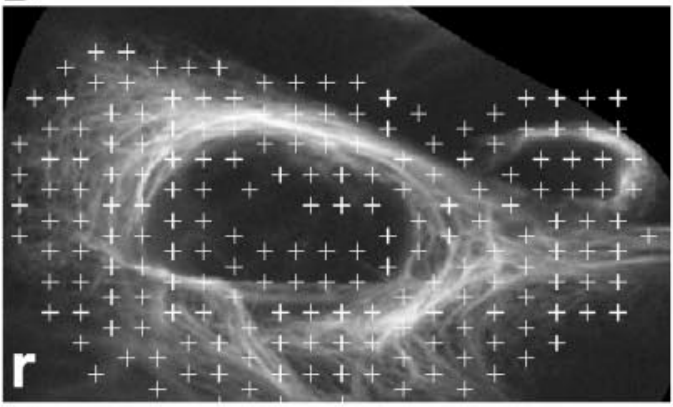

C

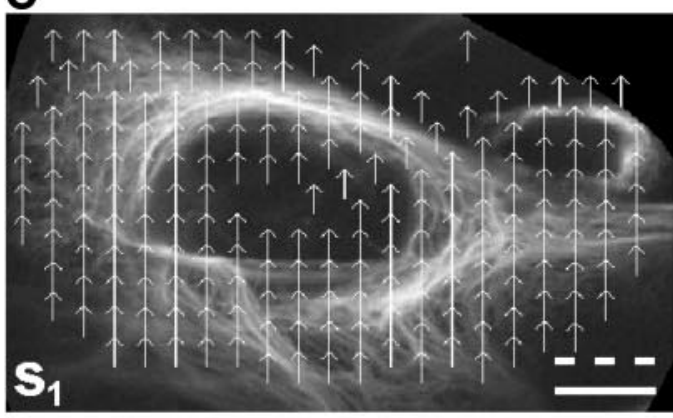

D
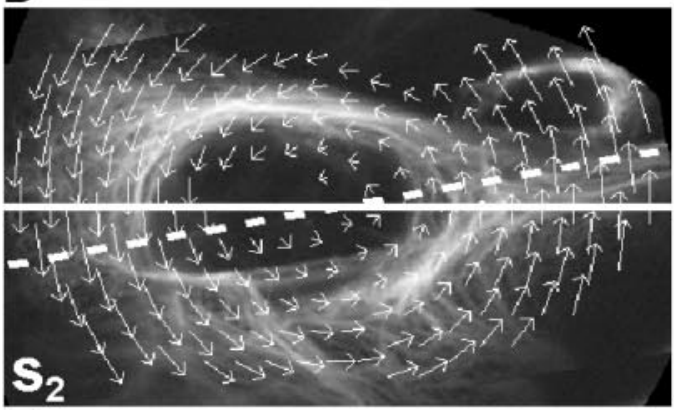

E

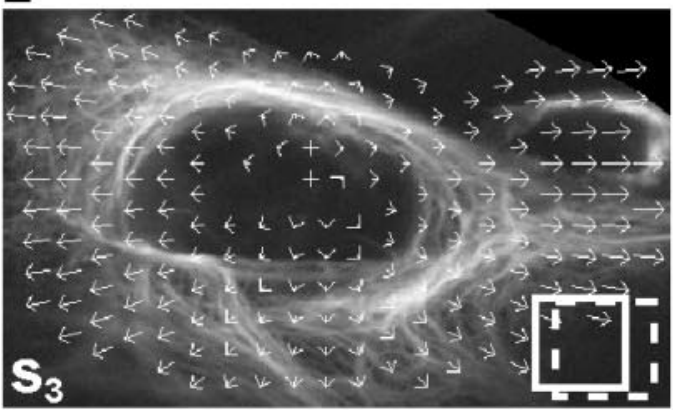


RMSE of 0.06. Note that a relatively large amount of pixels does not belong to the cytoskeleton but to the dark background. These pixels had similar feature vectors and, therefore, could hardly be distinguished. Matches found in these areas were usually considered invalid during backmatching, which shows the importance of backmatching as a quality measure. Better results were obtained when more features with larger windows were used. When 12 features, 4 pyramid levels, and masking, median filtering, and interpolation of the disparity map were used, the percentage of matched points increased to $69 \%$, with a concomitant decrease of the RMSE to 0.0001 . Although $69 \%$ can be considered as a relatively low percentage of matched pixels, the remaining $31 \%$ of all image pixels belong to the background or to the dark right and left upper corner. When the program was forced to match only pixels lying on the cytoskeleton, the percentage of matched points increased to $100 \%$. For all matching described further in this study, 12 features, 4 pyramid levels, and masking, median filtering, and interpolation of the disparity map were used.

Program testing results. Translation of the reference image over a vertical distance of 20 pixels (Fig. $1 C$ ) had virtually no affect on the results: the percentage of matched image points remained $69 \%$, whereas the RMSE slightly increased to 0.51 . The coordinates found by HFVM and the expected coordinates deviated by no more than two pixels.

After $10^{\circ}$ rotation of the reference image (Fig. $1 D$ ), the program retrieved $66 \%$ of all image points with a RMSE value of 2.15. The increase in the RMSE is reflected in an increase in the deviation of the matching coordinates found by HFVM from the expected coordinates, e.g., $82 \%$ deviated by $\leq 2,15 \%$ by $3-4$, $2.5 \%$ by $5-6$, and $0.5 \%$ by $7-8$ pixels.

Artificial 2-D deformation, e.g., 10\% stretching in horizontal direction and 5\% stretching in vertical direction, with the center of the image remaining at the same place (Fig. $1 E$ ), did not affect the percentage of matched points: of all image points, $67 \%$ could be matched. The RMSE, however, increased to 8.82 as reflected in the deviation of the matching coordinates found by HFVM from the expected coordinates. Although only $66 \%$ of the matched pixels deviated by $\leq 2$ pixels from the expected coordinates, the remaining $34 \%$ deviated by $\geq 3-4$ pixels.

Spontaneous cytoskeletal movements. Seven images of the time series (images 1, 6, 11, 16, 21, 26, and 31; each separated from each other by $5 \mathrm{~min}$ ) were selected for matching with HFVM. Please refer to the Supplementary Material ${ }^{1}$ for this article (published online at the American Journal of Physiology-Cell Physiology web site) to view a movie of the total time-lapse series. In the first image of the time series, pixels belonging to cytoskeletal filaments were manually selected (total number: 2,968 pixels) and traced throughout the time

${ }^{1}$ Supplemental material to this article is available online at http:// ajpcell.physiology.org/cgi/content/full/283/2/C639/DC1.

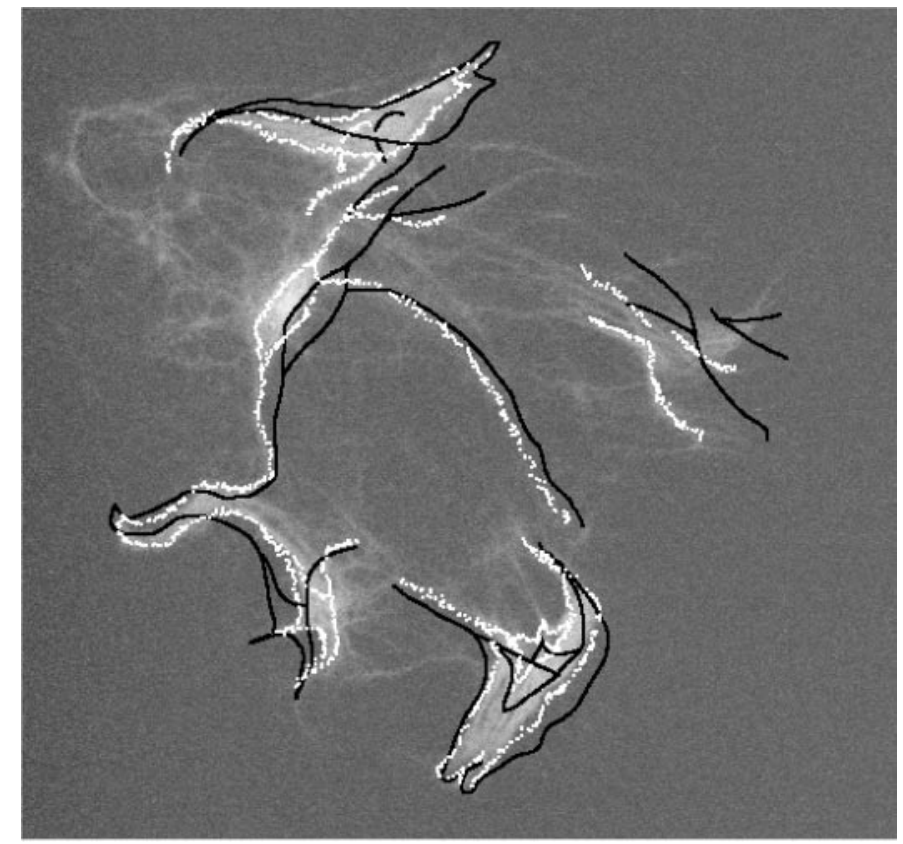

Fig. 2. Last image of a time series of 31 two-dimensional images of the spontaneous movement of the cytoskeleton, obtained at the central level of a cell (image interval $60 \mathrm{~s}$ ). Black dots indicate pixels belonging to cytoskeletal filaments (total of 2,968 points) as selected by hand in image 1, whereas white dots indicate matching results after application of HFVM on the first and last images of time series. A movie of the total time-lapse series can be viewed as Supplementary Material to this article.

series. Matching was performed between the first and second image, the second and third, and so on. Each time, the matching pixels were used as reference pixels for matching the next pair of images. Finally, the last image (image 31) was also matched directly to the first one, thereby skipping the intermediate matching.

By using intermediate matching, the final percentage of matched cytoskeletal pixels in image 31 (white pixels in Fig. 2) amounted to $98 \%$ of the originally selected pixels in image 1 (black pixels in Fig. 2). The loss of $2 \%$ undefined pixels occurred at random throughout the series. Skipping intermediate matching, e.g., comparing image 31 directly to image 1, did not influence the percentage of matched points. This finding indicates that HFVM is a robust procedure for retrieving matching pixels over several consecutive images or over relatively large distances. However, after reconstruction of the cytoskeletal filaments in image 31, they appeared much smoother after direct matching with the reference image 1 than after intermediate matching. Although the percentage of retrieved pixels was almost identical for both methods, intermediate matching apparently led to small discretization errors. This resulted in a less accurate reconstruction of cytoskeletal displacement, e.g., the matched structures did not always coincide with the filaments (Fig. 3).

The integrated horizontal $x-y$ displacement field after 30 min of acquisition time is presented in Fig. 4. The displacement is depicted in the number of pixels, 

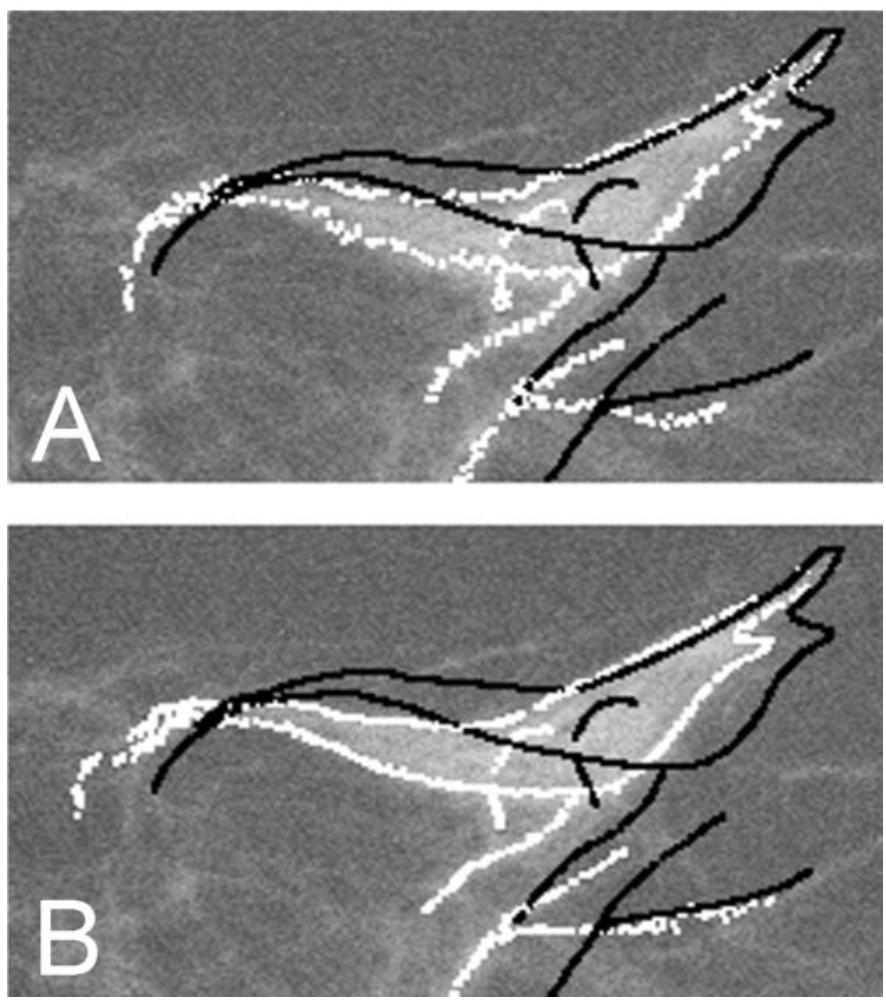

Fig. 3. Detail of matching results of Fig. 2 with $(A)$ and without $(B)$ intermediate matching.

with each pixel representing a $0.05-\mu \mathrm{m}$ distance. With a total cell width of $\sim 25 \mu \mathrm{m}$, the maximal displacement detected within the plane of view was $2.1 \mu \mathrm{m}(42$ pixels), $\sim 10 \%$ of the cell width. In the nuclear area, no displacement could be detected because this structure contains no GFP-vimentin. The area around the nucleus showed hardly any displacement, whereas the maximum displacement was noticed near the cell boundaries. Also, from the movie of the total time-lapse series, it becomes obvious that the vimentin filaments surrounding the nucleus have hardly any motility, whereas, in the cytoplasm, wavelike motions of these

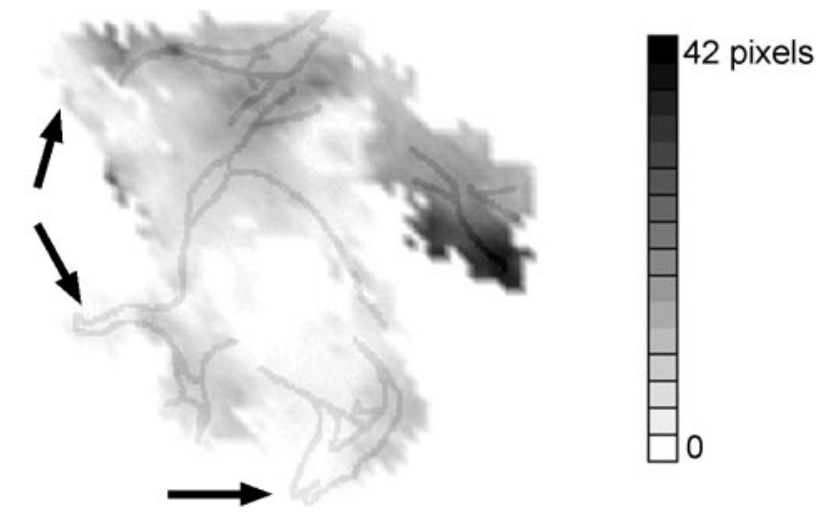

Fig. 4. Total displacement field after $30 \mathrm{~min}$ for same cell as in Fig. 2 . One pixel shift corresponds to $0.05 \mu \mathrm{m}$. Arrows indicate nonmoving spots at the cell boundary, suggesting that these represent focal adhesion points. filaments can be observed. Certain spots at the cell boundary (see arrows in Fig. 4) do not move, suggesting that these represent focal adhesion points.

The disparity maps can also be used to estimate the cytoskeletal deformation. Figure 5 shows the linear fiber strain along the intermediate filaments plotted as gray level on the original location. Fiber strain $\epsilon_{f}$ is calculated by using the disparity map from the matching process between images 1 and 31 of the time series and is defined as

$$
\epsilon_{f}=\frac{l-l_{\text {ref }}}{l_{\text {ref }}}
$$

in which $l$ and $l_{\text {ref }}$ denote the fiber length in images 31 and 1 , respectively. Fiber strain along the fiber direction varied from -0.5 to 0.5 . Regions with shortening within the elapsed $30 \mathrm{~min}$ are alternated with regions with lengthening. Whether this estimated fiber strain reflects real shortening or lengthening remains questionable, because fibers may have changed their orientation from in-plane to out-of-plane or vice versa, resulting in apparent shortening or lengthening. Because only two dimensions of in-plane disparities were estimated, this question remains unsolved.

\section{DISCUSSION}

This is the first report describing the application of a HFVM program on the natural displacement of the cytoskeleton of living cells. By applying HFVM to images of stably transfected CHO-K1 cell lines expressing a GFP-vimentin hybrid protein, we have shown that, with proper parameter and configuration settings, HFVM can recognize and trace $60-70 \%$ of all image points in artificially translated, rotated, or deformed images. If only points belonging to the cytoskeleton are selected for matching purposes, the percentage of matched points increases to $98 \%$. This high percentage of recognition is also reached in a time series of consecutive images, despite an inevitable certain degree of bleaching of the fluorescence over the recording time of $30 \mathrm{~min}$. Apparently, bleaching does not affect feature values. In these images, HFVM enables the detection as well as the quantification of spontaneous cytoskel-

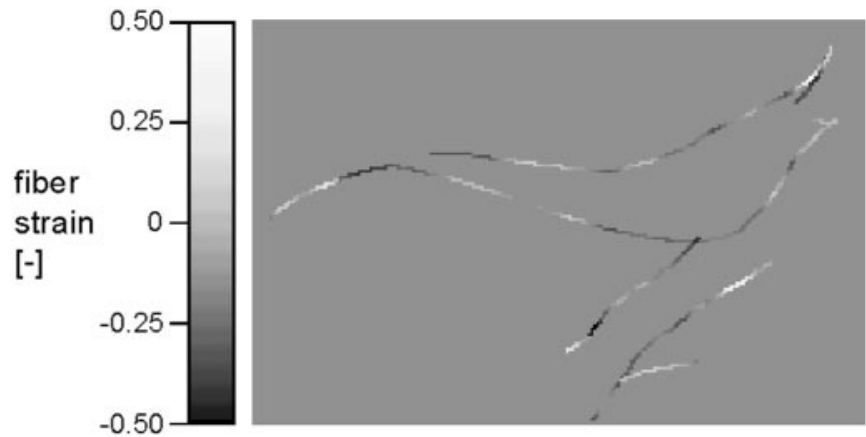

Fig. 5. Linear fiber strain along the intermediate filaments plotted as gray level on the original location. Fiber strain is calculated by using the disparity map from the matching process between images 1 and 31 of the time series and is defined as $\left(l-l_{\text {ref }}\right) / l_{\text {ref }}$, in which $l$ and $l_{\text {ref }}$ denote local fiber length in images 31 and 1 , respectively. 
etal movements of up to $10 \%$ of the cell width. Therefore, HFVM appears to be a reliable method for quantifying the displacements of the vimentin cytoskeleton in living cells.

Intermediate filaments (IFs) like vimentin are known to undergo dynamic changes in distribution and organization during cell growth, polarization, and differentiation $(6,11,17)$. In GFP-vimentin-expressing cells, IFs were observed to change curvature and orientation over the course of several minutes $(7,11)$ and to exhibit wavelike motions (8). Quantitative characterization of the experimental system, i.e., specimen geometry and deformation, is as important on the microscopic scale of the cell as it is for macroscopic tissue testing. However, to date, there is no report providing quantitative descriptions of cytoskeletal deformations. Because conventional image analysis programs using areal correlation methods fail to derive this information, we implemented $\operatorname{HFVM}(12,13)$.

There are several major advantages of using GFPvimentin and the HFVM program to study the mechanical behavior of the cytoskeleton. First, use of GFPvimentin enables the visualization of the entire cytoskeleton, in contrast to the spatially very limited information derived by using fluorescent-labeled microspheres attached to the cell surface $(1,10)$. Moreover, in contrast to microspheres, GFP-vimentin allows the determination of cytoskeletal shape and deformation in living cells without making any contact with them. Also, compared with microinjection of fluorescent vimentin or vimentin antibodies, GFP-vimentin is less hazardous to cells and allows the study of vimentin localization under more physiological conditions. HFVM overcomes the problem that conventional image analysis programs, using areal correlation methods, have with even small rotational movements. Because HFVM is based on multiresolution image matching by using a number of features and pyramid levels, it renders detailed information over the deformation of the entire cytoskeleton, either being spontaneous or caused by external loading. Conventional image programs cannot extract this detailed information. Another advantage of HFVM is that it is less affected by differences in intensity values because, in our study, it takes into account 11 extra characteristics of the pixel and its surroundings. The next advantage of HFVM is the increased speed of the matching process. If matching is started at the top level of the pyramid with low spatial resolution, a rough indication of the actual deformation can be obtained. The estimated disparity map can be used as input initial disparity map for matching the next lower level of the pyramid. If this is done, the search areas in this lower level of the pyramid are indicated with a higher precision because they are located immediately around the expected search pixel. This will speed up and improve the matching process because, at the lowest pyramid level, with the highest spatial resolution, matching has to be performed only in very localized areas. Even when the matching process is not sped up by the use of multiple pyramid levels, computational time will only be in- creased by $\sim 33 \%$ using four pyramid levels $(25 \%+$ $6.25 \%+1.56 \%$ ). When 12 features, 4 pyramid levels, and masking, median filtering, and interpolation of the disparity map are used, computational time for matching images of $768 \times 512$ pixels on a Sun workstation was $<1 \mathrm{~min}$.

Our results also show that preselection of points belonging to the cytoskeleton to be matched resulted in significantly better matching results than when trying to match all image points. This seems trivial because, if no preselection has been performed, a large number of image points to be matched have an identical background gray scale intensity and, hence, almost identical feature vectors. However, although preselection improves matching results, the selection of image points to be matched by hand is a time-consuming effort.

In our time series of 31 images, the reconstructed cytoskeletal filaments in image 31 appear much smoother after direct matching with reference image 1 than after intermediate matching. Every matching process in itself inevitably leads to very small discretization errors. Therefore, in the first matched image, the pixels found might already be a little bit off the actual cytoskeletal fiber. If this pixel is taken as reference pixel for the next search, the discretization error is likely to be enlarged. This finally results in a less accurate reconstruction of cytoskeletal displacement, e.g., the matched structures do not always coincide with the filaments (Fig. 3). However, because matching is possible even in the case of $10 \%$ strain and rotation, this discretization error can be overcome in almost all circumstances by immediately matching to the original reference image.

Although a certain degree of bleaching of the fluorescence over a recording time of $30 \mathrm{~min}$ is inevitable, this did not influence the percentage of recognized, matched pixels. This may be explained by the fact that bleaching does not affect feature values.

Confocal microscopy allows visualization with a very high spatial resolution in the $x-y$ direction and a high spatial resolution in the $z$ direction. The benefits of this are obvious for the $x$ and $y$ axes. For the $z$ direction, however, this high resolution might be counterproductive because slight cytoskeletal movements might already result in out-of-focal plane movement. This might hamper the matching process, although, from our analysis of the time series of spontaneous movements, this problem did not appear to be prominent. If, on the other hand, cells are exposed to external loading, the issue of out-of-plane movement might have a much higher importance. In this case, future research has to be focused on 3-D reconstruction, which is, however, somewhat limited by the resolution in the $z$ direction, which is approximately four times less than the resolution in the $x$ and $y$ direction.

The results shown in Fig. 5 also underscore the importance of 3-D disparity estimation. Although the 2-D disparity maps allow homogeneous strain analysis for the entire cytoskeleton, out-of-plane movements, fiber buckling, and changes in fiber orientation from in-plane to out-of-plane or vice versa cannot be de- 
tected with our current method. Therefore, for the moment, we can only reflect to fiber strain as apparent lengthening, shortening, or shear.

One may wonder to what extent the vimentin-GFP images represent a cytoskeletal structure with physiological behavior. Because the GFP-vimentin construct is driven by a highly active CMV-promotor, we must take into account the possibility that the vimentin intermediate filaments might be overexpressed. In addition, formation of a morphological normal vimentin cytoskeleton appears to be temperature sensitive. A recent study by Reichenzeller et al. (15) has shown that correct GFP-vimentin incorporation into the preexisting cytoskeleton only occurs at reduced $\left(\leq 30^{\circ} \mathrm{C}\right)$ temperatures. Because in our study the cells are allowed to grow at $30^{\circ} \mathrm{C}$ for a certain period of time, it is likely that we have studied cells with a normal cytoskeletal organization. In addition, GFP-vimentin-transfected cells exhibit a mechanical behavior that might be different from nontransfected cells. Therefore, image analysis results of GFP-vimentin-transfected cells only show the mechanical behavior in the presence of GFPvimentin. Conclusions with respect to mechanisms of mechanotransduction have to be made with some reluctance.

Because of their low solubility in solutions of physiological ionic strength and the lack of evidence for significant pools of soluble units in vivo, IFs traditionally have been regarded as static cytoskeletal structures. However, IFs and their constituent proteins show remarkable plasticity during many physiological activities, including cell division, axon outgrowth in neurons, and myogenesis in muscle cells. HFVM enables us to quantify this dynamic behavior of IFs.

\section{APPENDIX}

Names, meaning, filter kernel coefficients, and weight of the 12 digital filters used to calculate the feature values are as follows.

Var55 represents local $5 \times 5$ variance, a statistical feature that was also used as a mask for feature vector matching (only pixels with a local variance exceeding threshold 5 were matched); weight 2 .

Average5 represents local $5 \times 5$ average; filter kernel coefficients $(1,1,1,1,1 ; 1,1,1,1,1 ; 1,1,1,1,1 ; 1,1,1,1,1$; $1,1,1,1,1) / 25$; weight 2 .

H5 and V5 represent first-order horizontal and vertical derivatives; filter kernel coefficients $(2,1,0,-1,-2) / 4$; weight 4.

H7 and V7 represent small-scale second-order horizontal and vertical derivatives; filter kernel coefficients $(1,0,-1,0$, $1,0,-1) / 2$; weight 4 .

H13 and V13 represent medium-scale second-order horizontal and vertical derivatives; filter kernel coefficients $(2,1$, $0,-1,-2,-1,0,1,2,1,0,-1,-2) / 8$; weight 2 .

H19 and V19 represent large-scale second-order horizontal and vertical derivatives; filter kernel coefficient $(-3,-2,-1$, $0,1,2,3,2,1,0,-1,-2,-3,-2,-1,0,1,2,3) / 8$; weight 4 . Gauss77 represents Gaussian $7 \times 7$ filter; filter kernel coefficients $(1,1,1,1,1,1,1 ; 1,1,1,2,1,1,1 ; 1,1,3,5,3,1$, $1 ; 1,2,5,8,5,2,1 ; 1,1,3,5,3,1,1 ; 1,1,1,2,1,1,1 ; 1,1,1$, $1,1,1,1) / 84$; weight 4 .
Real7_3 represents edge detector; filter kernel coefficients $(-5,-5,-5,-7,-7,-6,-6 ;-5,-2,3,-4,-12,-9,-6$; $-5,3,27,28,-3,-12,-7 ;-7,-4,28,59,28,-4,-7 ;-7$, $-12,-3,28,27,3,-5 ;-6,-9,-12,-4,3,-2,-5 ;-6,-6$, $-7,-7,-5,-5,-5) / 100$; weight 4 .

We thank Ingrid Groenenberg and Viviane Heijnen for technical support and Barbie Machiels for preparation of the GFP-vimentin construct.

\section{REFERENCES}

1. Barbee KA, Macarak EJ, and Thibault LE. Strain measurements in cultured vascular smooth muscle cells subjected to mechanical deformation. Ann Biomed Eng 22: 14-22, 1994.

2. Chien KR, Knowlton KU, Zhu H, and Chien S. Regulation of cardiac gene expression during myocardial growth and hypertrophy: molecular studies of an adaptive physiologic response. FASEB J 5: 3037-3046, 1991.

3. Chien S, Li S, and Shyy YJ. Effects of mechanical forces on signal transduction and gene expression in endothelial cells. Hypertension 31: 162-169, 1998.

4. Davies PF. Flow-mediated endothelial mechanotransduction. Physiol Rev 75: 519-560, 1995.

5. Frangos JA, Eskin SG, McIntire LV, and Ives CL. Flow effects on prostacyclin production by cultured human endothelial cells. Science 227: 1477-1479, 1985.

6. Goldman RD, Chou YH, Dessev C, Dessev G, Eriksson J, Goldman A, Khuon S, Kohnken R, Lowy M, Miller R, Murphy K, Opal P, Skalli O, and Straube K. Dynamic aspects of cytoskeletal and karyoskeletal intermediate filament systems during the cell cycle. Cold Spring Harb Symp Quant Biol 56: 629-642, 1991.

7. Heidemann SR, Kaech S, Buxbaum RE, and Matus A. Direct observations of the mechanical behaviors of the cytoskeleton in living fibroblasts. J Cell Biol 145: 109-122, 1999.

8. Ho CL, Martys JL, Mikhailov A, Gundersen GG, and Liem RK. Novel features of intermediate filament dynamics revealed by green fluorescent protein chimeras. J Cell Sci 111: 1767$1778,1998$.

9. Langille BL and O'Donnell F. Reductions in arterial diameter produced by chronic decreases in blood flow are endotheliumdependent. Science 231: 405-407, 1986.

10. Lee AA, Delhaas T, Waldman LK, MacKenna DA, Villarreal FJ, and McCulloch AD. An equibiaxial strain system for cultured cells. Am J Physiol Cell Physiol 271: C1400-C1408, 1996.

11. Martys JL, Ho CL, Liem RK, and Gundersen GG. Intermediate filaments in motion: observations of intermediate filaments in cells using green fluorescent protein-vimentin. $\mathrm{Mol} \mathrm{Biol} \mathrm{Cell}$ 10: 1289-1295, 1999.

12. Paar G, Kuijpers NHL, and Gasser C. Stereo vision and 3D reconstruction on a processor network. In: Machine Perception Applications, edited by Pinz A and Poelzleitner A. Graz, Austria: IAPR/TC8, 1996.

13. Paar G and Poelzleitner W. Robust disparity estimation in terrain modeling for spacecraft navigation. Proceedings of the 11th ICPR. International Association for Pattern Recognition, 1992.

14. Prahlad V, Yoon M, Moir RD, Vale RD, and Goldman RD. Rapid movements of vimentin on microtubule tracks: kinesindependent assembly of intermediate filament networks. J Cell Biol 143: 159-170, 1998.

15. Reichenzeller M, Burzlaff $A$, Lichter $\mathbf{P}$, and Herrmann $H$. In vivo observation of a nuclear channel-like system: evidence for a distinct interchromosomal domain compartment in interphase cells. J Struct Biol 129: 175-185, 2000.

16. Swynghedauw B. Molecular mechanisms of myocardial remodeling. Physiol Rev 79: 215-262, 1999.

17. Vikstrom KL, Lim SS, Goldman RD, and Borisy GG. Steady state dynamics of intermediate filament networks. J Cell Biol 118: 121-129, 1992.

18. Yoon M, Moir RD, Prahlad V, and Goldman RD. Motile properties of vimentin intermediate filament networks in living cells. J Cell Biol 143: 147-157, 1998. 